\title{
CIENCIAS HUMANAS Y PLURALISMO DE LA VERDAD ${ }^{1}$
}

\author{
HUMAN SCIENCES AND PLURALISM OF TRUTH
}

\author{
Juan Carlos Aguirre García \\ Universidad del Cauca \\ Diagonal 50 Norte, Transversal 18-744, Casa I-7 \\ Popayán, Colombia \\ jcaguirre@unicauca.edu.co
}

\begin{abstract}
RESUMEN
El ámbito de las ciencias humanas, debido a su indefinición, tiende a albergar posturas cercanas a la posverdad, sin siquiera someterlas a escrutinio. El propósito de este artículo se ubica en este contexto. La pregunta que lo guía es si el conocimiento en ciencias humanas puede despedirse o renunciar a la búsqueda de la verdad y, de ser así, si aún conserva su carácter de conocimiento, aunque relativo, subjetivo e, incluso, irracional. Se optará por una vía alterna, es decir, se defenderá que no es necesario caer en el relativismo, el subjetivismo o el irracionalismo en ciencias humanas y que es posible un concepto de verdad que salvaguarde
\end{abstract}

Este artículo es parte del proyecto de investigación "La objetividad en ciencias humanas: dificultades en su definición y polémicas recientes en torno a ella” (ID 5094), ejecutado por el grupo de investigación Fenomenología y Ciencia, y financiado por la Universidad del Cauca. Agradezco los valiosos aportes de los evaluadores del artículo, que se acogieron de manera plena. 
su especificidad. Para ello, se reconstruirán algunas polémicas recientes sobre el realismo y el antirrealismo en ciencias humanas; posteriormente, se recurrirá a la propuesta del pluralismo de la verdad, especialmente a la versión denominada pluralismo por correspondencia; finalmente se concluirá mostrando cómo a partir de ahí se pueden superar ciertas tensiones que se vislumbran en las discusiones epistemológicas sobre las ciencias humanas.

Palabras clave: ciencias humanas, correspondentismo, epistemologia, pluralismo, verdad.

\section{Abstract}

Because of its lack of definition, the Human Sciences are prone to include views close to post-truth, without subjecting them to scrutiny. This paper explores the question of whether knowledge in Human Sciences could renounce the search for truth, and, if so, whether it would preserve its nature of knowledge despite it being relative, subjective, and irrational. I claim that it is not necessary to fall into relativism, subjectivism, or irrationalism in the Human Sciences and that a concept of Truth which preserves their specificity is possible. To support this claim, I begin by first reconstructing some recent polemics about realism and anti-realism in the Human Sciences. Second, I explore the Pluralist Theory of Truth, especially the Correspondence Pluralism. I conclude by showing how to overcome some tensions that we perceive in epistemological discussions about the Human Sciences.

Keywords: Human Sciences, Correspondentism, Epistemology, Pluralism, Truth. 


\section{INTRODUCCIÓN}

Las discusiones históricas sobre el estatuto de cientificidad de las ciencias humanas y, sobre todo, el cuestionamiento reciente de su pertinencia en la formación académica, hacen sospechar que aquello que se agrupa bajo esta denominación no tiene, en el mejor de los casos, límites definidos o, en el peor, alberga lo más disímil e, incluso, contradictorio. Por consiguiente, podría hablarse de las ciencias humanas como un espacio caracterizado por su indefinición. Para algunos, esto es un punto a favor de ellas, toda vez que se atrincherarían en los intersticios para repeler los embates de una racionalidad omnicomprensiva respaldada en el arsenal matemático; para otros, en línea con los anteriores, las ciencias humanas abordarían el resto, lo no visto por las ciencias naturales o sociales ${ }^{2}$. Sin embargo, esta indefinición podría también ser tomada por algunos como carencia de rigor u honestidad académica, charlatanería e inutilidad. En cualquier caso, la porosidad de sus márgenes y la falta de discusiones epistemológicas sistemáticas sobre las ciencias humanas hacen que sean un terreno fértil para albergar posturas subjetivistas, relativistas e, incluso, irracionales.

Tal vez la perspectiva actual que mejor condensa las mencionadas posturas es la denominada posverdad. Los ejemplos de posverdad se han tornado lugares comunes; la palabra misma se ha integrado a nuestro lenguaje cotidiano, al punto de verla como un auténtico problema con implicaciones socioculturales que exceden el orden epistémico. Sería hora de revisar cuáles son los ancestros de esta postura. Podría verse, por ejemplo, que muchas de las ideas que prefiguran este concepto han sido acogidas

2 Estas posturas encuentran respaldo en el estudio arqueológico hecho por Foucault en Les mots et les choses, donde establece que el origen de las ciencias humanas se da al margen de lo que llama el triedro de los saberes: las ciencias matemáticas y físicas, "las ciencias (como las del lenguaje, de la vida, de la producción y la distribución de las riquezas)", y el "pensamiento de lo mismo". La alusión tanto a los intersticios y al resto, pretende condensar esta intuición foucaultiana. En efecto, el autor menciona explícitamente que "es en el intersticio (l'interstice) de esos saberes, más exactamente en el volumen definido por sus tres dimensiones donde [las ciencias humanas] encuentran su lugar” (358). Podría ser esta una razón de su indefinición. 
y difundidas, de manera amplia y militante en el contexto de las ciencias humanas. Si bien tal ejercicio es necesario, no se constituye en el objetivo de este texto. El propósito más que ofrecer alternativas al problema de la posverdad, en cuanto problema sociocultural, es explorar, en un contexto estrictamente epistémico, algunas discusiones recientes en las ciencias humanas, en aras de indicar su cercanía o lejanía con la posverdad; pero, sobre todo, identificar alternativas para bloquear el influjo de la posverdad en la epistemología de las ciencias humanas y exponer un concepto de verdad que no solo satisfaga la especificidad de su objeto, sino que conserve el estatuto de su cientificidad.

Para este propósito, se revisan algunas tesis que han hecho carrera dentro de las ciencias humanas y, como tales, parecen ser paradigmáticas. La mayoría de ellas se toman del trabajo del hermeneuta italiano Gianni Vattimo. Posteriormente, se evalúan estas tesis desde el denominado nuevo realismo, en especial, desde la propuesta de Maurizio Ferraris y Markus Gabriel. Si bien, en esencia, muchas de estas ideas, y sus respectivos argumentos y contraargumentos, fueron consideradas en el marco de la filosofía de la ciencia posterior a Thomas Kuhn, la originalidad del debate consiste en que se incrusta en el que ha sido considerado por muchos como el método de las ciencias humanas por antonomasia, la hermenéutica ${ }^{3}$. El

En este artículo se parte de la idea de que parece haber un consenso, aunque no unanimidad, entre los investigadores en que las ciencias humanas se diferencian de las naturales (no tan amplio es el consenso respecto de su distinción con las ciencias sociales) tanto en sus objetos de estudio como en sus métodos. En lo que tiene que ver con el método, los fundadores de la filosofía de las ciencias humanas (piénsese, por ejemplo, en Dilthey, Rickert o Windelband) reaccionaron en contra del monismo metodológico que se imponía debido al éxito de la ciencia natural. Tal y como lo afirma Georg von Wright, aquellos filósofos fueron llamados idealistas, aunque podría llamárseles mejor hermeneutas. Según von Wright, “el filósofo e historiador alemán Droysen parece haber sido el primero en introducir una dicotomía metodológica que ha tenido gran influencia; para ello, acuñó los términos explicación y comprensión" (5). De acuerdo con esta distinción, los métodos de la ciencia natural tendrían como función explicar la naturaleza, mientras que las ciencias humanas se esforzarían por comprender asuntos que exceden el alcance de la explicación. Por lo menos en la fase de confrontación con las ciencias naturales, los hermeneutas se esforzaron por elaborar una teoría de la comprensión que validara, aunque con otros presupuestos, los conocimientos propios de las ciencias humanas. Por lo tanto, Gadamer afirma 
segundo apartado defiende una alternativa a la dicotomía entre realismo y antirrealismo en ciencias humanas, a saber, el pluralismo de la verdad, propuesta defendida, especialmente, por Crispin Wright y Michael Lynch; sin embargo, se apuesta por una versión refinada del mismo, esto es, la expuesta por la profesora Gila Sher, conocida como el pluralismo por correspondencia. Obviamente, el tratamiento dado a ambas propuestas será esquemático, pues la sola exposición de una de ellas excedería los límites de este artículo. Se concluye mostrando cómo podría conservarse el carácter plural de la verdad sin caer en la tentación del relativismo; pero, más que eso, que la búsqueda de la verdad en ciencias humanas no es una discusión superada, sino que exige una respuesta ante el desafío de la posverdad.

\section{2. ¿ADIÓS A LA VERDAD EN CIENCIAS HUMANAS?}

El concepto verdad tiene una larga tradición en la historia del pensamiento, por lo que una definición unívoca puede ser un esfuerzo destinado al fracaso. Desde la definición de Aristóteles, según la cual “Decir, en efecto, que el Ente no es o que el No-ente es, es falso, y decir que el Ente es y que el No-ente no es, es verdadero" (1011b 25), ha corrido mucha agua bajo el puente. Para Nicolás y Frápolli, "la verdad es uno de los temas centrales de la Filosofía del Conocimiento, de la Filosofía del Lenguaje y de la Filosofía de la Lógica, y su ámbito alcanza hasta la Ontología y la Filosofía Práctica” (9). En efecto, los autores dividen su compilación Teorías contemporáneas de la verdad en

que la hermenéutica puede entenderse como "teoría de la comprensión" (112). De una manera más amplia, Grondin define la hermenéutica como "una reflexión metodológica sobre la pretensión de verdad y el estatuto científico de las ciencias del espíritu. Esta reflexión destaca sobre el trasfondo del éxito que han conocido las ciencias puras desde el siglo XIX, éxito en buena medida atribuido al rigor de sus métodos, aspecto en que las ciencias del espíritu se muestran muy deficitarias. Si las ciencias del espíritu quieren llegar a ser ciencias respetables, deben apoyarse en una metodología que la hermenéutica debe poner al día” (18). Es en este sentido restringido que se afirma en este artículo que la hermenéutica ha sido el método de las ciencias humanas por antonomasia, dejando a un lado las variaciones históricas que ha sufrido la hermenéutica o los elementos técnicos que albergan sus discusiones. 
clases de teorías: de la correspondencia, prooracionales, fenomenológicas, hermenéuticas, coherenciales, pragmáticas e intersubjetivistas. Esto indica que la verdad no es una cuestión simple de abordar, no solo por la cantidad de campos que abarca, además, por las diversas perspectivas desde las que se considera; asimismo, es un indicativo de la dificultad de abordar el concepto desde una única perspectiva, como de la necesidad de aproximarse a él considerando diversos enfoques (incluso aquellos que prima facie parezcan inconmensurables).

Las ciencias humanas, en tanto disciplinas, son posteriores a las ciencias naturales. En un principio, el concepto de verdad (que a primera vista parece estar en consonancia con los postulados básicos de una teoría correspondentista) se asumió, imitativamente, de estas ciencias; no obstante, una vez que se tomó en serio la labor de fundamentación de las ciencias humanas, el concepto de verdad comenzó a tornarse problemático. Si bien las ciencias humanas aspiraban a constituirse como ciencias, su objeto de estudio parecía distinguirse del objeto de las ciencias naturales; por ende, los métodos y los resultados tendrían que ser distintos.

Según Dilthey, las ciencias humanas ocuparían "esa otra mitad del globus intellectualis" (40), la cara dejada de lado por las ciencias naturales. En esta epistemología inicial de las ciencias humanas -tradición que no se ha extinguido, como veremos a continuación- estarían, por un lado, los hechos que pertenecen a la "estructura espacial y a la divisibilidad de la materia, tanto como a la necesidad mecánica, a la que está sometida la acción de la parte individual de ella” (49); por otro, estarían los hechos del espíritu, que se fundan en "la unidad de la conciencia y la espontaneidad de la voluntad" (49). La contradicción entre ambos globos de la realidad dificulta la subsunción de las ciencias humanas en las naturales, por lo que aquellas tendrán que utilizar métodos diferentes a estas y, en consecuencia, los resultados difieren radicalmente:

Las regularidades que se pueden establecer en la esfera de la sociedad son muy inferiores en número, importancia y precisión formal a las leyes que han podido formularse acerca de la naturaleza, sobre la base segura de las relaciones 
espaciales y las propiedades del movimiento [...] Las ciencias de la sociedad no pueden permitir tal satisfacción del entendimiento. (Dilthey 84)

La alusión a la antigüedad del concepto de verdad, así como a las complejidades que encierra y, sobre todo, al terreno en disputa que se abre con la introducción de las ciencias humanas en el contexto de las disciplinas, tiene como fin mostrar que las discusiones recientes sobre la verdad, de una de las cuales se dará cuenta en este apartado, no aparecen por generación espontánea, sino que se articulan con una tradición discursiva que hunde sus raíces en lo más preciado de la reflexión humana; solo que ahora, con el desafío de la posverdad, parecen tornarse urgentes de respuesta.

Como aporte a estas discusiones recientes podemos mencionar el libro de Gianni Vattimo, Addio alla verità, publicado en 2009. Este adiós se une a otras despedidas que conservan cierto aire de familia, por ejemplo, el Farewell to Reason de Paul Feyerabend o el "Farewell to Objectivity" de Ernst von Glasersfeld. En la introducción a su libro, Vattimo explica así el sentido del título: "es una despedida de la verdad como reflejo 'objetivo' de un 'dato' que, para ser descrito de forma adecuada, debe fijarse como estable, es decir, como 'dado'” (16). De acuerdo con esto, Vattimo está confrontando una visión de la verdad por correspondencia, que se puede caracterizar a partir de las siguientes cláusulas, tomadas de Noah Lemos: "una proposición es verdadera si y solo si se corresponde con los hechos [...] Una proposición es falsa si y solo si falla al corresponderse con los hechos" (9). Es importante adicionar una cláusula que a menudo agregan los defensores de la teoría de la verdad por correspondencia, toda vez que muestra de manera más clara el concepto que confronta Vattimo: "la verdad de una proposición o creencia es dependiente de los hechos o del modo como es el mundo" (Lemos 9).

El adiós a la verdad de Vattimo tendría que precisarse, entonces, como adiós a la verdad por correspondencia. El autor es claro en afirmarlo: aludiendo a autores como Maquiavelo, Gramsci y Lukács, sostiene que ellos "no alcanzan a ver que la verdad que vale la pena en política, así como en todos los demás campos, no es la correspondencia objetiva sino el horizonte 
paradigmático dentro del cual toda correspondencia es verificable" (Vattimo 17). Esta cita permite clarificar la verdad que confronta y, a la vez, la verdad que propone o que "vale la pena":

Hoy, pues, con mucha más claridad que en el pasado, la cuestión de la verdad es reconocida como una cuestión de interpretación, de puesta en acción de paradigmas que, a su vez, no son "objetivos" (ya que nadie los verifica ni falsifica, salvo basados en otros paradigmas), sino que es un tema de consenso social. (Vattimo 18)

La verdad que propone Vattimo estaría enmarcada en lo que se conoce en epistemología como constructivismo o construccionismo social del conocimiento. Paul Boghossian, crítico agudo del relativismo de este tipo de propuestas, reconstruye esta postura de la siguiente manera:

La verdad de una creencia no está relacionada con cómo son las cosas en una "realidad que existe de manera independiente"; asimismo, su racionalidad no dependería de que pueda ser corroborada de acuerdo con "procedimientos trascendentes de escrutinio racional". Más bien, el que una creencia sea o no conocimiento estaría en función, al menos en parte, del entorno social y material contingente en el que haya sido producida (o sostenida). (Boghossian 22)

Según esto, Vattimo propone un adiós a la verdad por correspondencia y una bienvenida a la verdad como construcción social; esta verdad no dependería de los hechos-evidencias que la justifiquen, sino que su legitimidad depende del paradigma compartido que respalda la interpretación; con otras palabras, la verdad es una cuestión de consenso social. La principal razón para la adopción de esta nueva perspectiva sobre la verdad tendría que ver con las posibilidades que abre para el "diálogo social e intercultural" (Vattimo 17), así como su consonancia con la pluralidad que caracteriza a la sociedad contemporánea. Más aún, Vattimo afirma que "el adiós a la verdad es el inicio, y la base misma, de la democracia" (18). Incluso, emulando a Richard Rorty, quien habla de fundar la solidaridad humana 
no en la objetividad, sino, al contrario, la objetividad en la solidaridad (41), Vattimo da un paso más: "La parábola de la noción de verdad en el siglo XX se configura como una transición de la verdad a la 'caridad'” (19).

Para los propósitos de este artículo, es importante resaltar que Vattimo establece una distinción entre las ciencias naturales y las humanas. De cierta manera, concede que la noción de verdad por correspondencia opera en las primeras, mas no en las segundas: "Ahora bien, esto [de la verdad por correspondencia] puede hacerse en las ciencias que 'no piensan', ya sea porque no ponen en cuestión el horizonte (paradigma) dentro del cual se mueven, ya sea porque ignoran la totalidad de las relaciones dialécticas que condicionan a sus objetos" (16). Es claro que Vattimo salvaguarda su posición haciendo referencia tácita a Heidegger, para quien "la ciencia no piensa y no puede pensar [...] La ciencia no piensa" (8). Tal vez Vattimo no comparta la misma condescendencia con esas ciencias que parece haber en Heidegger, esto es, mostrar que está bien que no piensen pues esta actitud les ha permitido seguir adelante y obtener éxito.

La verdad como construcción social no opera tanto en las ciencias naturales sino en las humanas. Esto sugeriría que no hay una verdad, sino que, por lo menos, habría dos: una correspondiente con los hechos, propia de las ciencias naturales, y otra como construcción social o interpretativa - para conservar el espíritu de uno de los Fragmentos póstumos de Nietzsche: "No hay hechos, solo interpretaciones", que se presenta como telón de fondo de este debate- propia de las ciencias humanas. A primera vista, Vattimo estaría adoptando una concepción pluralista fuerte de la verdad; sin embargo, antes de considerar el pluralismo de la verdad, es preciso confrontar la propuesta de Vattimo en el mismo terreno en que la ubica: las ciencias humanas.

Si bien las teorías construccionistas han sido ampliamente debatidas y refutadas en el contexto de la filosofía de las ciencias naturales e, incluso, en las sociales, en el ámbito de las ciencias humanas la discusión es más bien reciente. El esfuerzo más consistente a este respecto es el denominado nuevo realismo, bautizado así por los filósofos Maurizio Ferraris y Markus Gabriel. En tanto no compete a este escrito exponer o criticar el nuevo 
realismo, en lo que sigue expondré las críticas más relevantes que plantean los neorrealistas a posturas como las de Vattimo.

En primer lugar, Ferraris coincide con Vattimo en que la discusión sobre la verdad no se restringe a la esfera epistémica, sino que tiene hondas implicaciones políticas; no obstante, a diferencia de la esperanza emancipadora de Vattimo, Ferraris ve cumplidas las profecías posmodernas en el creciente populismo mediático:

Los últimos años, en efecto, han enseñado una amarga verdad. Y esta es que el primado de las interpretaciones sobre los hechos, la superación del mito de la objetividad, se ha cumplido, pero no ha tenido los resultados de emancipación profetizados por los profesores. (42)

Debido a esto, la posmodernidad no fracasa porque sus objetivos sean inalcanzables; de lo que se trata es que estos objetivos se cumplieron, mas no produjeron el resultado esperado, a saber, la emancipación; al contrario, configuraron un nuevo espacio de manipulación: "El mundo verdadero ciertamente ha llegado a ser una fábula, es más [...] ha llegado a ser un reality, pero el resultado ha sido el populismo mediático, un sistema en el cual (con tal que se tenga el poder para ello) se puede pretender hacer creer cualquier cosa" (42). Todo lo contrario al fortalecimiento de la democracia que preveía Vattimo con el adiós a la verdad.

El abandono de la verdad por correspondencia tiene que ver con el estatus dado a los hechos ${ }^{4}$. En el caso de Vattimo, los hechos no existen, a menos que sean determinados de acuerdo con un paradigma compartido; en ese caso, la crudeza de los hechos debe matizarse con las comillas: no se

Así como se requiere actualizar las discusiones sobre la verdad en las ciencias humanas, también es preciso poner en cuestión, desde la perspectiva de estas ciencias, el concepto de hecho; más específicamente, si es posible la objetividad en las ciencias humanas (ver a este el respecto el artículo de Aguirre). No se indica con ello que no se puedan considerar de manera independiente (Gaukroger, por ejemplo, plantea que algunos teóricos no abordan el problema de la objetividad desde la verdad, sino desde la justificación); de lo que se trata es de considerar la posibilidad de explorar modos de relación entre objetividad y verdad. 
trata de hechos sino de hechos. Este desplazamiento, muy usual en discusiones epistemológicas, es denominado por Ferraris ironización (en igual sentido se había pronunciado décadas atrás el filósofo David Stove a partir de lo que llamó la neutralización de las palabras de éxito). Es justamente contra esta tesis negacionista de los hechos que se alza el nuevo realismo.

Tanto Ferraris como Gabriel coinciden en afirmar que ha sido Kant quien, al establecer la distinción entre cosa en sí y cosa tal y como se nos aparece o se constituye en nuestra subjetividad (fenómeno), dio patente de corso al construccionismo. Gabriel, por ejemplo, plantea que "el constructivismo se basa en la suposición de que no existen hechos en sí, que somos nosotros quienes construimos todos los hechos mediante nuestros diversos discursos o hechos científicos” (11). Según Gabriel, "el representante más importante de esta tradición es Immanuel Kant, quien decía que no podemos conocer el mundo tal como es en sí mismo. Sea lo que sea lo que conozcamos, siempre es algo elaborado por seres humanos" (11). Asimismo, Ferraris ve la obra de Kant como un camino natural que conduce al construccionismo posmoderno: "He aquí el origen de la posmodernidad. Siguiendo y radicalizando a Kant, los construccionistas confundirán sin dejar residuos (es decir, aboliendo también el noúmeno) la ontología con la epistemología, lo que hay (y que no depende de esquemas conceptuales) y lo que sabemos (y que depende de esquemas conceptuales)" (74-5).

La estrategia usada por Ferraris es doble: en primera instancia, distingue los términos de la dupla ser-saber, que parecen confundir a los construccionistas. El primer término de la dupla estaría representado por la ontología y tendría como características ser inenmendable, la postulación del mundo externo, y el carácter no necesariamente lingüístico, histórico y teleológico, así como finito de la experiencia. El segundo término correspondería a la epistemología y sus características serían la enmendabilidad, la postulación del mundo interno (interno a los esquemas conceptuales), y el carácter lingüístico, histórico, libre, infinito y teleológico de la ciencia (Ferraris 84).

En segunda instancia, Ferraris parece calcar lo ya logrado por Searle en La construcción de la realidad social, en especial, lo referido a la distinción 
entre hechos brutos y hechos institucionales; los primeros son el objeto de estudio de las ciencias naturales (ontología objetiva), los segundos lo son de las ciencias sociales (ontología subjetiva). Si bien es cierto que hechos como el matrimonio, el dinero o los Estados dependen de los humanos (son hechos institucionales), otros hechos (brutos) como las montańas, el agua o las lunas de Júpiter son independientes de nosotros. Ferraris lo plantea de manera gráfica:

El construccionista sostiene que si el fuego quema, el agua moja y la pantufla está en la alfombra, esto depende de esquemas conceptuales. Está claro que no es así. Depende del hecho de que el fuego queme, el agua moje y la pantufla esté en la alfombra, son caracteres ontológicos, no epistemológicos. (82-3)

Además de coincidir con Searle en lo tocante a la ontología, Ferraris también suscribe que es posible investigar objetivamente tanto en la ontología objetiva como en la subjetiva:

Por ejemplo, es un hecho que en 1813, en Leipzig, el contingente sajón abandonó a Napoleón y se puso del lado de los austríacos, prusianos, rusos y suecos; es un evento que puede ser evaluado de varios modos, pero es de todos modos un hecho, y quien pretendiese decir que no tuvo lugar no daría una mejor interpretación de lo acontecido, sino que simplemente diría una cosa falsa. (106)

Una vez establecidas estas distinciones, Ferraris se propone formular lo que llama un tratado de paz perpetua entre las intuiciones construccionistas y realistas:

1. Los objetos naturales son independientes de la epistemología y hacen verdaderas las ciencias naturales.

2. La experiencia es independiente de la ciencia.

3. Los objetos sociales son dependientes de la epistemología, sin ser por ello subjetivos. 
4. "Las intuiciones sin conceptos son ciegas" vale sobre todo para los objetos sociales (donde tiene un valor constructivo), y subordinadamente para el acercamiento epistemológico al mundo natural (donde tiene valor reconstructivo). 5. La intuición realista y la intuición construccionista tienen, pues, la misma legitimidad en sus respectivos sectores de aplicación. (Ferraris 123-4)

El punto 5 pareciera dejarnos en una situación similar a la que nos dejó Vattimo: es posible ser realista en las ciencias naturales y construccionista en ciencias humanas. Por lo tanto, al igual que Vattimo, Ferraris y Gabriel defienden una postura pluralista. Gabriel abiertamente lo dice: "mi propia posición es una forma de pluralismo, y estoy convencido de que tanto el monismo como el dualismo son demostrablemente falsos" (66). Su propia posición es denominada por él como campos de sentido; al respecto afirma:

la lógica moderna ha solapado casi por completo el concepto de ámbitos objetuales con la noción de cantidad. Sin embargo, no todas las áreas son conjuntos de objetos contables y matemáticamente descriptibles; esto no se aplica, por ejemplo, a las obras de arte o a los sentimientos complejos. No todas las áreas en las que aparece algo son áreas objetuales. Por lo tanto, el concepto de campo de sentido es más objetual. (75)

En aras de la precisión, Gabriel define dichos campos de sentido como "áreas en las que determinados objetos aparecen de una manera determinada [...] Dos campos de sentido pueden referirse a los mismos objetos, que aparecen en ellos de forma diferente" (77).

Pese a la aparente similitud, aún se conservarían algunos rasgos distintivos entre Vattimo y Ferraris y Gabriel. En primer lugar, a diferencia de Vattimo, es posible defender la existencia de los hechos, la independencia de estos respecto de la mente humana y su cognoscibilidad; en segundo lugar, hay hechos construidos socialmente; no obstante, estos pueden abordarse de manera objetiva, siendo susceptibles de valor de verdad; finalmente, una postura realista sobre los hechos es más favorable políticamente que una construccionista; en esta última, al no haber hechos sino hechos, la realidad 
(que en verdad sería realidad) no pasa de ser una construcción hecha a la medida de los intereses de los políticos, medios de comunicación y grupos de poder.

Estas diferencias, sin embargo, no parecen ser lo suficientemente relevantes, por lo que podríamos preguntar si se concedió demasiado en ese tratado de paz. El esfuerzo por defender la realidad de los hechos (naturales, brutos), tal vez no permitió a Ferraris tematizar los hechos (institucionales) de las ciencias sociales o humanas; es una victoria pírrica creer que la distinción entre ciencias naturales y sociales o humanas mermaría la avanzada construccionista. Si bien Ferraris es claro en mostrar, bajo su caracterización de lo que llama muyotrismo, que no hay una escisión sensata y responsable entre el mundo natural y el mundo social -en sus palabras, que "hay un hilo ininterrumpido que conduce del hecho de que la nieve es blanca si y solo si la nieve es blanca al hecho de que, en esa nieve, el 27 de enero de 1945, los soldados del Ejército Rojo entraron en Auschwitz, y de allí a la 'vergüenza que los alemanes no conocieron"' (107)-, nada permite ver en su exposición esa línea de continuidad; más aún, el punto 5 del tratado de paz, así como la tesis de los campos de sentido, sugerirían que, basta aceptar que hay hechos naturales, todo lo demás es susceptible de interpretación.

En lo que sí podríamos estar de acuerdo es en que la victoria, pese a ser pírrica, socavó la pretensión construccionista enarbolada por Vattimo, la que -en aras de un pluralismo- adoptó el monismo de creer que todo se reducía a simple construcción social. El logro de Ferraris y Gabriel fue postular un pluralismo que respetaba tanto el ámbito de lo natural como de lo social/humano. Pero ¿es sólido este pluralismo o es proclive a los mismos problemas del pluralismo epistémico? La respuesta a esta pregunta permitirá revisar, entre otras cosas, si la propuesta del nuevo realismo contribuye a una adecuada fundamentación epistémica de las ciencias humanas. 


\section{Aproximación al pluralismo de la Verdad}

Se vio que las dos posturas más relevantes en la epistemología contemporánea de las ciencias humanas respaldan alguna versión del pluralismo. El propósito de este apartado es considerarlas a la luz de algunas teorías pluralistas de la verdad, con el fin de mostrar sus aproximaciones y diferencias. Por lo menos en la presentación hecha hasta el momento, no parece haber un desarrollo sistemático de lo que los autores toman como pluralismo. Debido a esto, es preciso hacer las distinciones pertinentes.

Hay que hacer, sin embargo, una advertencia: este apartado está construido en un lenguaje distinto a las sensibilidades disciplinarias de los autores mencionados anteriormente. Mientras que Vattimo, Ferraris y Gabriel pertenecen a lo que se ha dado en llamar filosofía continental, la mayoría de las discusiones que se condensan aquí se han dado, y aún se dan, en el marco de lo que se denomina filosofía analítica. En una lectura ligera, este apartado podría dar la impresión de que se trata de una mezcla forzada sin punto en común; no obstante, la razón de recurrir a tradiciones distintas a las continentales es que en ellas se encuentran modos de abordar un problema que en la filosofía continental no se ha esclarecido lo suficiente o ha contribuido a polarizaciones insolubles. Debe quedar claro, sin embargo, que estas tradiciones se unen en la búsqueda de una concepción pluralista de la verdad que tenga implicaciones para el problema de la verdad en las ciencias humanas, sin importar si tal concepción se ha dado en un marco conceptual o en otro. En ocasiones, levantar puentes entre tradiciones puede ser una estrategia útil para ampliar los márgenes reflexivos en los que tendemos a encasillarnos.

Para aproximarnos a la concepción pluralista de la verdad podemos recurrir a Pedersen y Wright -en especial su artículo "Pluralist Theories of Truth"-, para quienes la intuición del pluralismo de la verdad es que hay muchas maneras de ser verdadero; de una manera más precisa, los pluralistas sostienen que el número de propiedades de verdad es mayor que uno, esto es, "que hay más de una propiedad de verdad". La orilla que confrontan es, entonces, la versión monista de la verdad, según la 
cual la verdad consiste en tener una propiedad. En el clásico ejemplo de la proposición 'la nieve es blanca', la verdad de la proposición depende de si hay una correspondencia entre ella y el hecho al que se refiere. Con otras palabras, la verdad depende de la propiedad: corresponderse con. Ahora bien, Pedersen y Wright distinguen entre dos tipos de pluralismo: moderado y fuerte. Según el primero, "hay más de una propiedad de verdad, algunas de las cuales son tenidas por todos los enunciados verdaderos"; según el segundo, "hay más de una propiedad de verdad, ninguna de las cuales es tenida por todos los enunciados verdaderos". Similar distinción se puede establecer entre el monismo fuerte y el moderado: para el monismo fuerte, "hay exactamente una propiedad de verdad, que es tenida por todos los enunciados verdaderos"; para el moderado, "hay una propiedad de verdad, que es tenida por todos los enunciados verdaderos".

La versión de pluralismo defendida por Vattimo es, sin lugar a duda, una versión fuerte: las propiedades de verdad varían de acuerdo con el dominio en el que se profieran: el correspondentismo para las ciencias naturales, el construccionismo para las ciencias sociales y humanas. Ambas, en principio, serían excluyentes (no obstante, una lectura radical de esta postura podría conducir a un monismo de la verdad, al postular como principio único la dependencia del enunciado con el paradigma), es decir, no se puede exigir la correspondencia a las ciencias humanas, aunque sí es tolerable este principio en las ciencias naturales. Ferraris y Gabriel, por su parte, parecieran defender un pluralismo moderado, aunque el quinto punto del tratado de paz perpetua, donde se legitima tanto la intuición realista como la construccionista en los respectivos sectores o dominios de aplicación, parecen ubicarlo en el mismo pluralismo fuerte de Vattimo. Igual ocurre con los campos de sentido de Gabriel.

El pluralismo es una teoría atractiva y parece estar en concordancia con el sentido común. Lynch expresa la situación pluralista de esta manera:

Tú y yo creemos en todo tipo de proposiciones: que la tortura es moralmente bárbara, que el árbol más alto en el patio de enfrente es un abeto, que dos veces dieciséis es treinta y dos. Sin embargo, pese a las obvias diferencias respecto del 
sujeto, ordinariamente asumimos que estos tipos de proposiciones -que van de la moralidad a lo misceláneo de la vida cotidiana- son igualmente susceptibles de ser correctos o incorrectos, o nos permiten vérnoslas bien o no con las cosas. En resumen, intuitivamente las tratamos como si fueran verdaderas. No obstante, cuando buscamos en el mundo los objetos y las propiedades que algunas de estas creencias supuestamente representan, quedamos perdidos. Respecto, por lo menos, de algunas de las proposiciones que creemos, parece que no hay objetos, propiedades o hechos -en síntesis, no hay realidad-con la cual se correspondan. (1)

Este tipo de situaciones cotidianas puede desembocar en distintas posturas respecto de la verdad, por ejemplo, unos se proponen tomar como verdaderos solo los enunciados que tengan correspondencia con los hechos $y$, en consecuencia, desechar como sinsentido o carentes de verdad aquellos que no tengan tal correspondencia; otros tienen como propósito distinguir planos del pensamiento, como la representación y los sentimientos, y suprimir la posibilidad de verdad o falsedad en ellos; finalmente, otros considerarán que todos los juicios y las creencias son aptos para la evaluación racional ya que todos se proponen representar la realidad, solo que algunos fallan en hacerlo. Esta variedad de posturas pone en riesgo la concepción de la verdad, pues, o bien solo se aplicaría en un dominio del pensamiento, o bien podría tolerarse como verdadero aquello que tenga sentido dentro de un dominio particular, así entre en contradicción con otros dominios. En cualquiera de los casos es problemático, pues habría restricciones en la búsqueda de la verdad o la verdad no podría superar el relativismo.

La crítica de Ferraris y Gabriel a Vattimo es legítima en tanto este se despide de la verdad por no encontrar satisfactoria la concepción de verdad por correspondencia. No obstante, ¿es legítimo este paso? En otras palabras, si bien la teoría de la verdad por correspondencia no está exenta de dificultades, ¿habría que abandonar la verdad como un todo? Esta postura implicaría eludir la cuestión, pues si la teoría de la verdad por correspondencia no da cuenta plenamente de situaciones cotidianas como las descritas por Lynch, por ejemplo, morales o matemáticas, esto no dispensa de la 
búsqueda de una concepción de verdad que las involucre. Podría objetarse que Vattimo ha hecho una propuesta de verdad que confronta y amplía el alcance del correspondentismo, a saber, el pluralismo. Sin embargo, valdría preguntarse si el pluralismo indicado por Vattimo se configura como una teoría consistente de la verdad.

Es preciso comenzar con una intuición que subyace al pluralismo que defiende Vattimo (y, en alguna medida, también Ferraris y Gabriel): no hay una verdad sino muchas. Esta postura podría describirse en los mismos términos que Lynch denomina al pluralismo alético simple: "la 'verdad' simplemente es ambigua, al modo que 'paso' o 'banco' son ambiguos; es decir, la palabra comporta diferentes conceptos o significados en diferentes contextos. Algunas veces, 'verdad' significa 'correspondencia' [...]; otras veces significa 'supergarantía”' (54). Los postulados que subyacen al pluralismo alético simple son "que hay más de un concepto de verdad; que hablar de verdad es equívoco; por tanto, que el significado de 'verdad' es, en un sentido, susceptible al contexto" (54). El último postulado es claro en Vattimo: al plantear que una creencia cuenta como conocimiento solo si está en función del entorno social contingente en el que se produce, el autor subordina la verdad de la creencia al contexto; asimismo, Vattimo identifica, por lo menos, dos tipos de verdad: el propio de las ciencias que, según él, no piensan (verdad por correspondencia) y la verdad que vale la pena, unida con el horizonte paradigmático; en este mismo sentido, el uso de la palabra verdad sería ambiguo. De acuerdo con lo anterior, Vattimo defendería un pluralismo alético simple.

No obstante su aparente continuidad con el sentido común, el pluralismo alético simple es vulnerable por muchos flancos. Lynch presenta cuatro objeciones. Si bien en conjunto socavan la consistencia del pluralismo alético simple, solo me referiré a la tercera de ellas, en tanto es, prima facie, la que más relación tiene con la versión de Vattimo; esta objeción tiene que ver con las generalizaciones que el concepto de verdad debe proporcionar (las otras objeciones son que no permite entender las inferencias mixtas y la validez; no permite comprender las proposiciones compuestas y no puede dar cuenta de los truismos normativos). En palabras de Lynch: "algo útil 
acerca del concepto de verdad es que permite que hagamos generalizaciones ciegas" (57).

Lynch toma como caso la proposición "Todo lo que Sócrates dijo era verdadero". Ahora bien, Sócrates dijo muchas cosas de distintos tipos, por ejemplo, "Solo sé que nada sé", o "mi esposa Jantipa tiene mal carácter", o "Nací en Atenas". Es claro que los tres enunciados podrían pertenecer a diferentes tipos de proposiciones, esto es, pertenecen a varios dominios. En ese caso, en la proposición inicial "Todo lo que Sócrates dijo era verdadero”, analizada en la generalidad de los casos, ¿en qué sentido es verdadera? Si respondemos que es verdadera en cualquiera de los sentidos en que una proposición particular sea verdadera, no se cumpliría en estricto sentido lo concerniente a la generalización. Tendría que entenderse la generalización como "Todo lo que Sócrates dijo era verdadero en un sentido u otro; esto es: que para cada proposición que Sócrates dijo, la proposición es o verdad 1, o verdad 2, o verdad 3, etc.” (57). No obstante, esta respuesta plantea dos problemas: en primer lugar, elude el propósito original de la generalización, que nada dice sobre una propiedad disyuntiva de las afirmaciones reales y posibles de Sócrates; en segundo lugar, el pluralista alético simple tendrá que estar de acuerdo en que también habrá una forma en que una proposición sea falsa; por tanto, tendrá que conceder que mucho de lo que dijo Sócrates era falso en algún sentido u otro. En este caso, también se elude el propósito de la generalización.

Puede verse, entonces, que el pluralismo alético simple, en cuanto teoría de la verdad, no ofrece respuestas plausibles a cuestiones como la generalización, centrales a la extensión del concepto de verdad; por tanto, no se puede convertir en una alternativa sólida a las teorías de la verdad que confronta. Versiones más sofisticadas de pluralismo, como el pluralismo reductivo de Crispin Wright, son, a la luz de Lynch, vulnerables a los mismos desafíos que socavan el pluralismo alético simple (Lynch 59-67). Por consiguiente, Lynch expone una teoría pluralista de la verdad que resista las críticas de los demás pluralismos y que denomina como teoría funcional de la verdad, que trata de unir dos intuiciones: que la verdad es una (one) y que la verdad es múltiple (many). 
Una teoría de la verdad tendría que responder a dos preguntas básicas: ¿qué es la verdad? y ¿en qué condiciones una proposición es verdadera? Antes de responder a la primera, Lynch considera el asunto de las condiciones. Para poder establecerlas, comienza con una serie de conocimientos obvios o de sentido común, denominados por él como truismos. De estos truismos, Lynch resalta tres: objetividad - "la creencia de que $p$ es verdadera si, y solo si, respecto de la creencia que $p$, las cosas son como se cree que son" (70)-; norma de creencia -"es prima facie correcto creer que $p$ si y solo si la proposición que $p$ es verdadera" (70) - y fin de la investigación - "siendo otras cosas iguales, las creencias verdaderas son una meta valiosa de la investigación" (70)-. Según el autor, estos truismos han dado a las teorías tradicionales de la verdad la esencia nominal a la verdad; por tanto, su teoría también parte de ellos.

El argumento central de Lynch consiste en sugerir que tales truismos no deben tomarse solo como propiedades de la verdad, sino como funciones que estas propiedades desempeñan. Por ejemplo, en el caso del truismo objetividad, este no debe tomarse

como algo que tenga que explicarse por algo más fundamental, sino como revelando un aspecto del rol funcional de la verdad. Estrictamente hablando, "retratar las cosas como ellas son" es parte del "rol de verdad" que hacen las proposiciones que tienen la propiedad de verdad. (Lynch 71)

De acuerdo con esto, la pregunta por las condiciones bajo las cuales una proposición es verdadera tendría que articular los truismos, entendidos en términos de propiedades y de funciones: "los truismos nos dicen que las proposiciones verdaderas son aquellas que tienen una propiedad que tiene una cierta función en nuestra economía cognitiva" (Lynch 71). En su exposición amplia, Lynch establece un sistema en el que integra no solo las proposiciones a los truismos básicos o nucleares, sino que establece unas características truistas (truish features), entre las cuales podrían estar la independencia de la garantía, la transparencia, la preservación de la verdad en la inferencia válida, la ambivalencia, la no contradicción. Estas 
características pueden ser esenciales o accidentales; por ejemplo, "ser un color es una característica esencial de la propiedad ser rojo; pero ser el color favorito de Tom es solo una característica accidental de ser rojo" (Lynch 74). Asimismo, son cuasi lógicas y explicativas, y dependen de los truismos nucleares, al punto de "constituir, al menos en parte, tanto la esencia real de la verdad como su esencia nominal” (Lynch 74).

Esta exposición le permite a Lynch defender que, en efecto, la verdad es una $\mathrm{o}$, con sus propias palabras, que "hay una sola propiedad y un solo concepto de verdad" (74). De manera explícita lo estipula: "La propiedad ser verdadero (o la propiedad de verdad) es la propiedad que tienen las características truistas esencialmente, o que juegan el rol de verdad como tal' (74). Esta definición salvaguarda las intuiciones monistas, al considerar que solo hay una propiedad de ser verdadero, es decir, rebate la pretensión del pluralismo alético simple que deja margen a la ambigüedad en el concepto de verdad. Ahora bien, ¿̨de qué manera Lynch defiende que la verdad es múltiple?

Para exponer mejor su propuesta pluralista, Lynch recurre a la filosofía de la mente, en especial, a la idea recurrente en esa disciplina según la cual "las propiedades mentales son múltiplemente realizables" (Lynch 69). Para ilustrarlo toma el caso del dolor en humanos, en animales no humanos y en el caso hipotético de los marcianos. En cada uno de los casos, la propiedad mental dolor se realiza de modo diferente, pues esta depende de la propiedad neural del organismo. En consecuencia, el que un organismo tenga dolor depende de las propiedades físicas de tal organismo; es por ello que afirma que "tener una de esas propiedades es lo que hace que tenga la propiedad sicológica relevante" (Lynch 69). Este caso es trasladado por Lynch al terreno de la teoría funcionalista de la verdad:

Parece plausible tratar la verdad -así como el dolor- como una propiedad superviniente: que si un contenido de creencia -una proposición- es verdadero está determinado, al menos en la mayoría de los casos, por tener alguna otra propiedad. Y esto es compatible con decir que hay más de una propiedad por la que esto puede ocurrir. (Lynch 70) 
Para poder justificar esta afirmación, sin echar por la borda lo logrado respecto del monismo, Lynch tiene que postular que "la verdad está [...] inmanente a propiedades ontológicamente distintas" (74). Su explicación a esta difícil cuestión es la siguiente:

Digamos que donde la propiedad F sea inmanente a, o manifestada por la propiedad $\mathrm{M}$, es a priori que las características esenciales de $\mathrm{F}$ son un subconjunto de las características de M. Puesto que es a priori que todas las características esenciales de la propiedad son un subconjunto de sus propias características, toda propiedad se manifiesta a sí misma. Así pues, la manifestación, como la identidad, es reflexiva; sin embargo, a diferencia de la identidad, es no simétrica. Donde M y F son propiedades ontológicamente distintas -individuadas por conjuntos no idénticos de características esenciales y relaciones-y M manifiesta F, F por tanto no manifiesta a M. (Lynch 74-5)

La consecuencia de esto es que existen propiedades distintas a la verdad; sin embargo, pueden manifestar la verdad siempre y cuando incluyan, dentro de sus propias características, algunas características de verdad. En el caso, por ejemplo, de la propiedad de una proposición como 'corresponderse con la realidad', se tiene que esta manifiesta la verdad; no obstante, esto ocurre si, de manera a priori, las características truistas son un subconjunto de las características de esa propiedad.

Podría resumirse lo básico de la propuesta de Lynch de la siguiente manera: la verdad es múltiple porque diferentes propiedades pueden manifestar la verdad en distintos dominios de investigación; ahora bien, la verdad es una porque hay una sola propiedad manifestada, y verdad nombra rígidamente esa propiedad (Lynch 78).

Antes de relacionar la propuesta pluralista de Lynch con el contexto de las ciencias humanas, es preciso mencionar por lo menos una crítica a esta postura, con el único propósito de no descartar la intuición correspondentista a la hora de abordar el pluralismo de la verdad; es decir, que la posibilidad del pluralismo para las ciencias humanas no implica, como lo hace Vattimo, descartar la verdad por correspondencia. Para ello mencionaré brevemente 
algunas críticas y la propuesta de Gila Sher, quien formula un pluralismo correspondentista.

En líneas generales, la crítica de Sher consiste en que Lynch respalda su teoría de la verdad en principios universales que se toman como obviedades (truismos), sin la debida justificación; de igual modo, más que dar cabida a las propiedades tradicionales (correspondencia, justificación ideal, correspondencia causal) como realizadores de la verdad, Sher sugiere que las formas de correspondencia son mejores candidatos para ello. Ahora bien, la autora no asume un concepto tradicional de correspondencia, sino que lo caracteriza de un modo amplio:

$(C O R)$ La verdad es un asunto de conexiones sustantivas y sistemáticas entre el lenguaje y el mundo. Estas conexiones ocurren en un caso particular si, y solo si, ese aspecto de la realidad del que trata un enunciado o una teoría es, directa $\mathrm{o}$ indirectamente, y basado en algunos principios pertinentes (de acuerdo con el tipo de caso), como el enunciado o la teoría dicen que es. (Sher 159-60)

Según Sher, la verdad surge en la intersección de tres características o condiciones del pensamiento humano: inmanencia, trascendencia y normatividad. La primera tiene que ver con pensar directamente sobre el mundo, sobre alguna faceta del mundo, o sobre algo en el mundo; la segunda consiste en tomar una perspectiva externa al pensamiento interno que estamos analizando, esto es, "ascender a un meta-lenguaje o moverse hacia una teoría de trasfondo" (Sher 160). Finalmente, la normatividad tiene que ver con el estándar para responder afirmativamente a la pregunta por la verdad, a saber, ¿̇este pensamiento inmanente está a la altura de la realidad?

Como puede sospecharse, Sher duda no solo de respaldar la teoría en truismos, sino de postular una definición de verdad; según ella, en tanto la verdad es una cuestión sustantiva y compleja, que juega un papel importante en muchas áreas de la vida humana y es objeto de múltiples intereses, se requiere una teoría sustantiva de la verdad que esté abierta a la posibilidad de una red compleja de principios, tal y como los esbozados. 
Si bien exponer la propuesta del pluralismo correspondentista excede los intereses de este artículo, es preciso mencionar, por lo menos, ciertos aspectos. En primer lugar, la propuesta de Sher considera esencial el carácter correspondentista de la verdad: "hay un sentido en que no podemos alejarnos de la verdad por correspondencia, no importa a dónde vayamos" (162). En segundo lugar, lo relevante no es la pregunta sobre si todos los enunciados verdaderos se conectan con la realidad de la misma manera, sino si "los principios que subyacen a la correspondencia en un área son sistemáticamente distintos de los de otras áreas" (162). Siendo la matemática su campo de interés, Sher ejemplifica con la distinción entre verdad en física y verdad en matemática: "Supongamos que, en física, la referencia (un constituyente importante de la referencia), está basado, en gran medida, en una relación causal directa entre expresiones físicas y objetos en el mundo. ¿Debe la referencia en matemática basarse en una relación causal directa entre el lenguaje matemático y el mundo?" (162) Si bien parece obvio que no, esto no se puede determinar desde los truismos. Por ende, Sher plantea un elemento que me parece central en su propuesta, a saber, que el teórico de la verdad tiene que trabajar mano a mano con el especialista en un área de investigación, de modo que pueda comprenderse en conjunto el modelo de correspondencia de dicha área.

A diferencia de la teoría tradicional de la correspondencia, en sus distintas versiones (pictórica, metáfora del museo, visión isomórfica), la teoría de Sher no toma la verdad como una cuestión que tiene que ver exclusivamente con cómo es el mundo o que la verdad sea completamente independiente de la mente; el asunto es, más bien, respecto de una verdad que trata con el modo en que significativamente el mundo es y cómo el mundo es significativamente independiente de la mente. Quedaría por determinar si esta teoría es, en últimas, pluralismo o, por el contrario, no es más que una forma débil de correspondentismo. Sin embargo, como se mencionó al inicio del apartado, estas versiones no son mutuamente excluyentes. En todo caso, la consideración de una teoría de la verdad como más que la búsqueda de "un algoritmo para descifrar la ruta de correspondencia en 
todos y cada uno de los casos y enunciados verdaderos de nuestro lenguaje" (Sher 178), da pie a una teoría abierta al trabajo interdisciplinario que se proponga "identificar y explicar los principios centrales de correspondencia, mostrar cómo están conectados en principio, demostrar su adecuación en ejemplos bien elegidos, y responder a objeciones pertinentes" (Sher 178).

En este apartado se consideró la teoría funcional de la verdad, una de las propuestas más sólidas respecto del pluralismo de la verdad; a su vez, se mencionó una versión de pluralismo que salvaguarda la correspondencia. A continuación, presentaré algunas posibles implicaciones de estas propuestas en el marco de las ciencias humanas.

\section{Ciencias humanas y pluralismo de la verdad}

El planteamiento de Vattimo respecto de la verdad condensa una crítica legítima a los modelos que la reducen a un modo limitado de correspondencia; no obstante, el planteamiento de Ferraris y Gabriel denuncia que de la crítica de Vattimo no se sigue el abandono de la verdad, esto es, que es posible una salvaguarda de su carácter plural que preserve una concepción consistente de ella. El recorrido hecho en el apartado anterior da cuenta del intento de algunos teóricos de la verdad por responder a este desafío. No obstante, algunos sectores de las ciencias humanas parecen haber claudicado ante tales posibilidades, no tanto por la dificultad que esto implica, sino por considerar que ya no son marcos teóricos adecuados para configurarse como disciplinas alternativas. Sin una discusión rigurosa de la verdad, estas ciencias quedarían vulnerables a posturas insostenibles, por ejemplo, a la descrita por Frankfurt como bullshit. Ciertamente, este autor afirma que

La proliferación contemporánea del bullshit también tiene fuentes más profundas en diversas formas de escepticismo que niegan que podamos tener un acceso confiable a una realidad objetiva y que, por tanto, rechazan la posibilidad de saber cómo son las cosas en realidad. Estas doctrinas antirrealistas debilitan 
la confianza en el valor de los esfuerzos desinteresados para determinar qué es verdadero y qué es falso e, incluso, en la inteligibilidad de la noción de investigación objetiva. (193)

Ahora bien, como lo sugiere la discusión del apartado anterior, el carácter plural de la verdad no se agota en la obviedad de considerar que hay distintos dominios de objetos epistémicos. Es claro que no es igual el dominio de los objetos físicos que el dominio de los objetos matemáticos, o de los objetos estéticos o morales. El auténtico reto consiste en proponer maneras desde las que los enunciados de dichos dominios adquieran sentido y trasciendan la mera opinión de quienes los formulen. La reflexión sobre el pluralismo de la verdad nos permite otear, por lo menos, dos rutas para responder a ese reto desde las ciencias humanas: en primer lugar, más que ver superadas las discusiones epistemológicas y, debido a ello decirles adiós, es preciso que las ciencias humanas se planteen una revisión constante de sus fundamentos epistémicos, incluso el más querido de la prioridad de las interpretaciones sobre los hechos. Aunque suene escandaloso, es posible coincidir con Lorraine Daston en que "todavía no hay una epistemología de las humanidades" (27). En segundo lugar, tal y como lo propuso Sher, este trabajo debe realizarse interdisciplinariamente: investigadores en epistemología y ciencias humanas que se propongan resolver preguntas sobre la verdad.

En el punto específico del pluralismo de la verdad expuesto por Lynch y matizado por Sher, se puede ver que esta propuesta no sucumbe al subjetivismo, relativismo o irracionalismo; más aún, abre vías de escape a las voces de sirena del bullshit que parecen conducir ineludiblemente a la posverdad (véase a este respecto la investigación de James Ball). La preocupación persistente en las ciencias humanas por no quedar reducidas a las pretensiones del naturalismo encuentra un respaldo sólido respecto de la pluralidad de dominios; no obstante, esto no implica una reclusión de la verdad a cada dominio de enunciación -que a la larga contradiría aspectos propios de la definición misma de verdad y abrazaría posturas, cuando menos, relativistas-, sino que implicaría, por lo menos, un llamado 
a buscar aquellas propiedades o principios que trasciendan cada dominio y, en razón de ello, continuar hablando, sin ambigüedad irresoluble, de verdad.

Finalmente, si, tal y como lo expuso Lemos, "alguna versión de la teoría de la verdad por correspondencia es correcta” (10), y, al menos en lo que se planteó del pluralismo/monismo fuerte/moderado y lo considerado sobre Sher no es totalmente incompatible el pluralismo de la verdad con alguna versión del correspondentismo, es plausible responder a Vattimo que no se trata de despedirse de la verdad, sino, al contrario, de darle la bienvenida en el contexto de las ciencias humanas, en aras de beneficiarse de las discusiones sobre las teorías de la verdad y, a la vez, de aportar en la ruptura de visiones que dejan por fuera su especificidad discursiva.

\section{Conclusión}

En este artículo me propuse considerar si era necesario que las ciencias humanas renunciaran a la búsqueda de la verdad o se plegaran a las pretensiones de una versión radical de correspondencia. El primer apartado permitió ubicar la discusión en el contexto de tales ciencias que se inscribe en la macro polémica entre realismo y antirrealismo. Se concluyó que realistas y antirrealistas en ciencias humanas defienden un pluralismo de la verdad. Sin embargo, se distinguió entre posturas que salvaguardan algunos principios subyacentes a la verdad y otras que renuncian deliberadamente a ellos. El segundo apartado bosquejó dos teorías pluralistas de la verdad, en las que se percibe cómo es posible defender el pluralismo sin abandonar algunos principios básicos, esto es, sin renunciar a una discusión razonada. El último apartado recogió algunas ideas para animar el trabajo interdisciplinario entre epistemólogos e investigadores de las ciencias humanas, en aras de la construcción sistemática de una epistemología en estas ciencias. 


\section{Bibliografía}

Aguirre, Juan. "La posibilidad de la objetividad en ciencias humanas". Cinta de Moebio, n. ${ }^{\circ}$ 67, 2020, pp. 1-13, www.cintademoebio.uchile.cl.

Aristóteles. Metafísica. Madrid: Gredos, 2013.

Ball, James. Post-Truth. How Bullshit conquered the world. Londres: Biteback, 2017.

Boghossian, Paul. El miedo al conocimiento. Contra el relativismo y el constructivismo. Madrid: Alianza, 2009.

Daston, Lorraine. "Objectivity and Impartiality. Epistemic virtues in the Humanities". The Making of the Humanities, Volume III: The Modern Humanities. Editado por Rens Bod, Jaap Maat y Thijs Weststeijn. Ámsterdam: Amsterdam UP, 2014, pp. 27-42.

Dilthey, Wilhelm. Introducción a las ciencias del espiritu. Madrid: Revista de Occidente, 1966.

Ferraris, Maurizio. Manifiesto del nuevo realismo. Madrid: Biblioteca Nueva, 2013.

Feyerabend, Paul. Farewell to Reason. Nueva York: Verso, 1988.

Foucault, Michel. Les mots et les choses. París: Gallimard, 1966.

Frankfurt, Harry G. "Sobre el concepto de bullshit". La importancia de lo que nos preocupa. Buenos Aires: Katz, 2006, pp. 171-94.

Gabriel, Markus. Por qué el mundo no existe. Barcelona: Pasado y Presente, 2017.

Gadamer, Hans-Georg. Verdad y método II. Salamanca: Sígueme, 2002.

Gaukroger, Stephen. Objectivity. A very short Introduction. Oxford: Oxford UP, 2012.

Glasersfeld, Ernst von. "Farewell to objectivity". Systems Research, vol. 13, n. ${ }^{\circ} 3$, 1996, pp. 279-86.

Grondin, Jean. ¿Qué es la hermenéutica? Barcelona: Herder, 2008.

Heidegger, Martin. What is called Thinking. Nueva York: Harper \& Row, 1968.

Lemos, Noah. An Introduction to the Theory of Knowledge. Cambridge: Cambridge UP, 2007.

Lynch, Michael. Truth as One and Many. Oxford: Clarendon Press, 2009. 
Nicolás, Juan y María Frápolli, editores. Teorías contemporáneas de la verdad. Madrid: Tecnos, 2012.

Nietzsche, Friedrich. Fragmentos póstumos (1885-1889). Volumen IV. Madrid: Tecnos, 2008.

Pedersen, Nikolaj y Cory Wright. "Pluralist Theories of Truth". The Stanford Encyclopedia of Philosophy, https://plato.stanford.edu/index.html.

_. $\quad$ Truth and Pluralism. Oxford: Oxford UP, 2013.

Rorty, Richard. Objetividad, relativismo y verdad. Escritos filosóficos I. Barcelona: Paidós, 1996.

Searle, John. La construcción de la realidad social. Barcelona: Paidós, 1997.

Sher, Gila. "Forms of Correspondence: the Intricate Route from Thought to Reality". Truth and Pluralism. Editado por N. Pedersen y C. Wright. Oxford: Oxford UP, 2013, pp. 157-79.

Stove, David. Popper and After. Four Modern Irrationalists. Oxford: Pergamon Press, 1982.

Vattimo, Gianni. Adiós a la verdad. Barcelona: Gedisa, 2010.

Wright, Crispin. Truth and Objectivity. Harvard: Harvard UP, 1994.

Wright, Georg von. Explanation and Understanding. Londres: Routledge \& Kegan Paul, 1971. 